\title{
Detection of Static and Dynamic Activities Using Uniaxial Accelerometers
}

\author{
Peter H. Veltink, Member, IEEE, Hans B. J. Bussmann, Wiebe de Vries, Wim L. J. Martens, \\ and Rob C. Van Lummel
}

\begin{abstract}
Rehabilitation treatment may be improved by objective analysis of activities of daily living. For this reason, the feasibility of distinguishing several static and dynamic activities (standing, sitting, lying, walking, ascending stairs, descending stairs, cycling) using a small set of two or three uniaxial accelerometers mounted on the body was investigated. The accelerometer signals can be measured with a portable data acquisition system, which potentially makes it possible to perform online detection of static and dynamic activities in the home environment. However, the procedures described in this paper have yet to be evaluated in the home environment. Experiments were conducted on ten healthy subjects, with accelerometers mounted on several positions and orientations on the body, performing static and dynamic activities according to a fixed protocol. Specifically, accelerometers on the sternum and thigh were evaluated. These accelerometers were oriented in the sagittal plane, perpendicular to the long axis of the segment (tangential), or along this axis (radial). First, discrimination between the static or dynamic character of activities was investigated. This appeared to be feasible using an rms-detector applied on the signal of one sensor tangentially mounted on the thigh. Second, the distinction between static activities was investigated. Standing, sitting, lying supine, on a side and prone could be distinguished by observing the static signals of two accelerometers, one mounted tangentially on the thigh, and the second mounted radially on the sternum. Third, the distinction between the cyclical dynamic activities walking, stair ascent, stair descent and cycling was investigated. The discriminating potentials of several features of the accelerometer signals were assessed: the mean value, the standard deviation, the cycle time and the morphology. Signal morphology was expressed by the maximal cross-correlation coefficients with template signals for the different dynamic activities. The mean signal values and signal morphology of accelerometers mounted tangentially on the thigh and the sternum appeared to contribute to the discrimination of dynamic activities with varying detection performances. The standard deviation of the signal and the cycle time were primarily related to the speed of the dynamic activities, and did not contribute to the discrimination of the activities. Therefore, discrimination of dynamic activities on the basis of the combined evaluation of the mean signal value and signal morphology is proposed.
\end{abstract}

\section{INTRODUCTION}

$\mathbf{T}$ O IMPROVE the rehabilitation treatment of patients, their activities of daily living should be evaluated in

Manuscript received July 12, 1994; revised October 7, 1996. This work was supported in part by the EUREKA project DYNAPORT of which McRoberts, The Netherlands, is the principal contractor and which is financed in part by the Dutch Ministry of Economic Affairs.

P. H. Veltink and W. de Vries are with the Institute for Biomedical Technology, University of Twente, 7500 AE Enschede.

H. B. J. Bussmann is with the Institute of Rehabilitation, Erasmus University, Rotterdam, The Netherlands.

W. L. J. Martens is with PhyVision bv, Gemert, The Netherlands.

R. C. van Lummel is with McRoberts bv, the Hague, The Netherlands.

Publisher Item Identifier S 1063-6528(96)09370-6. their domestic environments. This evaluation can give a good indication of the activity restrictions the patients experience because of their disabilities [1], [2]. The rehabilitation treatment can then be directed toward relieving these activity restrictions. The comparison of the daily life activities before and after a rehabilitation treatment enables the objective evaluation of the outcome of the treatment [2]. However, most objective instruments available to date measure impairment (e.g., reduced muscle force, reduced joint mobility) as opposed to activities actually performed, which are directly related to disability. Furthermore these instruments can only be used in the artificial environment of a laboratory or a doctor's office. Therefore, an ambulatory activity monitor is required for use in the home environment based on kinematic measurements using sensors mounted on the body.

In laboratory surroundings biomechanical and kinematic analyzes of activities are performed using instruments such as optokinetic measurement systems for measuring body movement and force plates for measuring ground reaction force. These measurement systems are important for thorough biomechanical analysis of joint moments and powers during body movements. They may be used to evaluate to what extent subjects are able to perform certain activities of daily living in a laboratory setting. However, these systems cannot measure to what extent these activities are actually performed in daily living in the domestic environment, which is important for an adequate assessment of disabilities [7].

Measurement of activities of daily living in a domestic environment requires a totally different approach and instrumentation. Measurements should not be limited to a small measurement space and should be performed over long periods of time (several hours to a day). The laboratorybound optokinetic systems and force plates do not satisfy these requirements. Small portable digital datalogger systems have become available in recent years. These systems can easily be worn at the waist and can acquire and store large amounts of data. Measurement of activities of daily living may become feasible using these systems if new and reliable measurement methods are developed on the basis of adequate sensors for registration of relevant kinematic quantities.

These sensors should be sufficiently small (on the order of a square centimeter) and light (a few grams) that they can be taped on the skin without introducing relative resonance movements. They should be easily mountable on the body by a clinician and stay reliably in place for the duration of the measurement. Their alignment should not be critical. They should be comfortable to the subject and not impede 
the activities of daily living. Therefore, they should not cross joints and only require short cables $(0.5 \mathrm{~m})$ for connection to the measurement unit. Their signals should contain maximal information about relevant kinematic quantities, and the number of sensors required for sufficient evaluation of daily life activities should be small.

Among the alternatives, seismic accelerometers satisfy these conditions as they are small and can be easily mounted on the skin. Signals from seismic accelerometers contain a component of the gravitational acceleration (giving information about inclination), and a component of the inertial acceleration of the sensor. In static situations, the accelerometer signal yields only inclination information, while in dynamic situations this information is combined with acceleration information [9], [10], [16]. Although many accelerometers are required for a full kinematic analysis of body movements [9], [10], [16], Willemsen et al. [17] showed that a single uniaxial accelerometer may be sufficient for the detection of swing and stance phases in gait.

Sensors, such as goniometers and switches or pressure sensors under the feet do not satisfy the conditions for monitoring activities of daily living in the domestic environment using a portable system, although they may provide useful additional information for activity monitoring [4], [13]. Goniometers are relatively large exoskeletal devices that cross the joints, which. may interfere with activities and move from their original placement. Foot switches and pressure sensors require long cables for connection to a portable unit, which is often worn at the waist.

The objective of this paper is to investigate the feasibility of distinguishing several static and dynamic activities using a small set of two or three uniaxial accelerometers placed on several body segments [15]. The considered activities are: standing, sitting, lying, walking, cycling, ascending and descending stairs. The detection scheme and sensor placement should be potentially suitable for real-time implementation in a portable system for monitoring activities of daily living in the domestic environment, although this will not be evaluated in this paper.

\section{THEORY}

\section{A. Static and Dynamic Activities in Relation to Body Topology}

Biomechanically, the human body can be considered to consist of a number of rigid bodies or body segments, linked together by the joints [5], [14]. In the case of a static activity (posture), the positions and orientations of the segments do not vary significantly with time. The static activities (e.g., standing, sitting, lying) can therefore be identified by the orientations of the segments with respect to the gravitational field. In the case of a dynamic activity, the positions and orientations of the segments do vary with time. Body movements occuring over distances which are large in comparison with the lengths of the body segments are most naturally achieved by moving the segments in a cyclical fashion [6]. Noncyclical movements are normally present only during short transitions between static and cyclical dynamic activities. Due to the topology of the linked chain of body segments, the cycle time of the

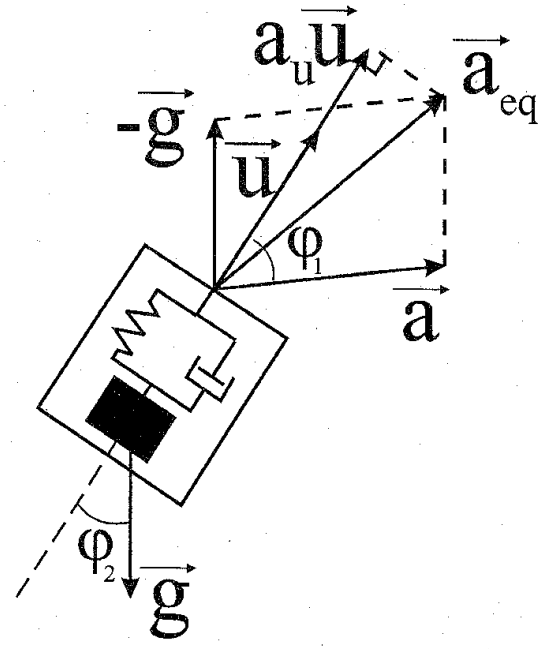

Fig. 1. Seismic uniaxial accelerometers measure the component $a_{u} \vec{u}$ of an equivalent acceleration $\vec{a}_{\mathrm{eq}}$ in the direction $\vec{u}$ of the sensitive axis of the accelerometer. This equivalent acceleration is the sum of the acceleration $\vec{a}$ of the sensor and the equivalent gravitational acceleration $\vec{g}$ acting on the seismic mass. $\varphi_{1}$ is the angle between the sensitive axis of the accelerometer and the acceleration $\vec{a} ; \varphi_{2}$ is the angle between the sensitive axis and the gravitational field.

trunk is $50 \%$ of the cycle time of each leg during symmetric cyclical movements.

\section{B. Kinematic Measurements by Means of Accelerometry}

Seismic uniaxial accelerometers measure the component $a_{u}(t)$ of an equivalent acceleration $\vec{a}_{\text {eq }}(t)$ in the direction of the sensitive axis of the accelerometer (Fig. 1)

$$
a_{u}(t)=\vec{a}_{\mathrm{eq}}(t) \cdot \vec{u}(t)
$$

$\vec{u}(t)$ being the unit vector in the direction of the sensitive axis of the accelerometer.

The equivalent acceleration $\vec{a}_{\text {eq }}(t)$ is the sum of the acceleration of the sensor $\vec{a}(t)$ and the equivalent gravitational acceleration $\vec{g}$ acting on the seismic mass (Fig. 1)

$$
\vec{a}_{\text {eq }}(t)=\vec{a}(t)-\vec{g} \text {. }
$$

The signal $a_{u}$ of a uniaxial accelerometer can therefore be expressed as

$$
\begin{aligned}
a_{u}(t) & =\vec{a}_{\mathrm{eq}}(t) \cdot \vec{u}(t) \\
& =a(t) \cos \left(\varphi_{1}(t)\right)-g \cos \left(\varphi_{2}(t)\right)
\end{aligned}
$$

with $a(t)$ being the magnitude of $\vec{a}(t), g$ the magnitude of $\vec{g}$, $\varphi_{1}(t)$ the angle between $\vec{a}(t)$ and $\vec{u}(t)$, and $\varphi_{2}(t)$ the angle between $\vec{g}$ and $\vec{u}(t)$.

It is not possible to distinguish a constant acceleration smaller than the gravitational acceleration $\left(9.81 \mathrm{~m} / \mathrm{s}^{2}\right)$ and a static inclination signal using the single uniaxial accelerometer. However, during the performance of normal dynamic activities the occurrence of a constant acceleration having a duration in the order of the cycle times of such activities is unlikely. Therefore, activities will be assimed to be dynamic if the uniaxial accelerometers mounted on the body yield a timevarying signal and static if the signal is constant. For static 


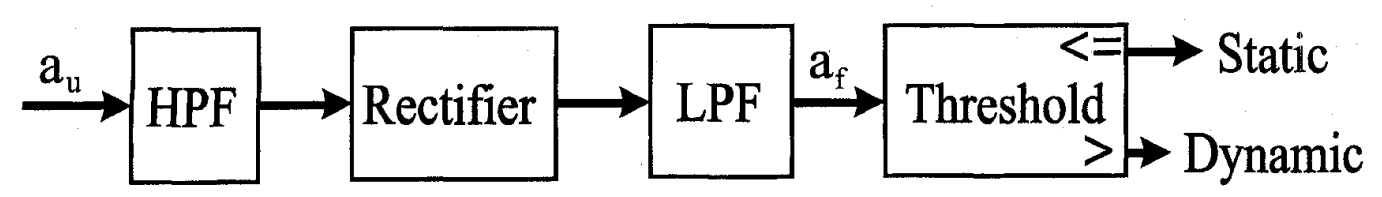

Fig. 2. Detector of the static or dynamic nature of activities. The signal $a_{u}$ of an uniaxial accelerometer mounted on the body is high-pass filtered, rectified and low-pass filtered to obtain a signal $a_{f}$ which is a measure of the time variation in the signal $a_{u}$. A static activity is detected if the signal $a_{f}$ is lower than a set threshold, otherwise an activity is deemed dynamic.

activities the constant accelerometer signal $a_{u}$ can be written as

$$
a_{u}=-g \cos \left(\varphi_{2}\right)
$$

The angle between the accelerometer axis and the gravity vector can be determined from the constant accelerometer signal, which gives information about the orientation of the acelerometer.

Note that detection of static activities on the basis of this assumption will only yield reliable results if the environment does not accelerate. It will not hold for instance when sitting in a car which moves in traffic, or in an elevator which accelerates or decelerates.

\section{Detection of the Static or Dynamic Nature of Activities}

On the basis of the assumption that dynamic activities yield time-varying and static activities constant accelerometer signals, the static or dynamic nature of activities can be detected by determining whether the signal varies with time. For this purpose we propose the detection scheme depicted schematically in Fig. 2: The signal of the tangential accelerometer on the thigh is high-pass filtered, rectified and subsequently low-pass filtered. High-pass filtering at a cut-off frequency of approximately $0.5 \mathrm{~Hz}$ will eliminate the current offset of the signal. Rectification and low-pass filtering at a cutoff frequency of approximately $0.1 \mathrm{~Hz}$ will yield a measure for the averaged signal deviation from the mean, weighted with an exponential time window, and is therefore a measure of "recent movement." The static or dynamic nature of activities can be discriminated by applying a threshold to the resulting signal. If the signal is above the threshold the activity is detected as being dynamic, while those below are deemed static. The threshold should be set such that small movements within static activities are not detected as dynamic, while still detecting all dynamic activities. It should be noted that the actual threshold choice is subjective.

\section{Distinguishing Static Activities}

If an activity has been detected as static, the type of static activity can be identified from the orientation of the body segments. These orientations can be obtained from the accelerometer readings which give only inclination information if the position and orientation of the body segments do not vary with time [see (4)].

The required number of accelerometers depends on the static activity. Standing, sitting and lying can be discriminated by observing the orientations of the trunk and the thigh only. This requires only one uniaxial accelerometer placed on each segment, oriented in the sagittal plane, perpendicular to the long axis of the segment (tangential), or along this axis (radial). Theoretically, a tangential thigh accelerometer and a radial trunk accelerometer should be sufficient to distinguish standing, sitting and lying (supine, prone, on a side). An additional accelerometer perpendicular to the sagittal plane is required to distinguish lying on the right from lying on the left side.

\section{E. Distinguishing Dynamic Activities}

Dynamic activities are normally achieved by cyclical movements [6] such as walking, ascending stairs, descending stairs and cycling. The accelerometer signals per cycle may differ in several aspects: morphology, mean, standard deviation and cycle time. These differences result from differences in geometric conditions imposed by the body topology, the environment and the speed of the activity. These differences may be used to distinguish between dynamic activities.

\section{METHODS}

\section{A. Experimental Methods}

Two sets of experiments were performed, in which ten male able-bodied subjects participated. Their age ranged between 23 and 42 years, height between 1.72 and $1.87 \mathrm{~m}$, and weight between 60 and $88 \mathrm{~kg}$. Discrimination between static activities was evaluated from the first set of experiments performed on five subjects (numbered 0-4). Detection of the static or dynamic character of activities and discrimination between dynamic activities was evaluated from the second set of experiments performed on the other five subjects (numbered 5-9).

The first group of five subjects (numbered $0,1,2,3$, and 4) performed static activities according to a fixed protocol: the subjects were requested to subsequentlly sit on a chair, stand, and lie on a bench (supine, prone, on right and left side). Each posture was held for approximately $10 \mathrm{~s}$. The subsequent static activities were repeated five times. Uniaxial accelerometers (ICsensors ${ }^{\mathrm{TM}} 5 \mathrm{~g}$, McRoberts) were mounted tangentially and radially on the mid-sternum and tangentially on the upper half of the thigh using double-sided tape (Fig. 3). The signals were acquired for subsequent off-line assessment using an IBM-compatible PC-AT, with an Analog Devices RTI-815 data acquisition system (12 bits). The accelerometer signals were preamplified (full scale was approximately \pm 5 g), filtered at $35 \mathrm{~Hz}$ (first order) and subsequently sampled at a rate of $100 \mathrm{~Hz}$.

The second group of five subjects (numbered 5, 6, 7, 8, and 9) performed both static and dynamic activities according to a set protocol. They were first asked to perform the static activities; sitting on a chair, lying on a bench (supine, prone, 


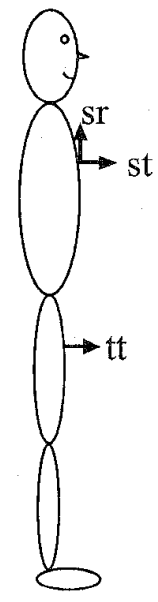

Fig. 3. Illustration of accelerometer mounting. Uniaxal accelerometers were mounted tangentially and radially on the sternum (st and $s r$ ) and tangentially on the thigh $(t)$. The sensitive axes were all directed in the sagittal plane.

on right and left side) and standing. Each activity was held for at least $5 \mathrm{~s}$ and repeated twice. Subsequently, the subjects were asked to perform several dynamic activities: walking (at slow, comfortable, and fast speeds), ascending stairs, descending stairs and cycling (at slow, comfortable and fast speeds). The dynamic activities were performed for a varying number of trials and a varying number of repetitions, and were alternated with periods of quiet standing (approximately $5 \mathrm{~s}$ ). Furthermore, the experiments contained parts of free movements (e.g., walking through a building from a walking session in the lab to an outdoor cycling session). For each person, the experiment, containing all the aforementioned activities, had a duration of approximately $30 \mathrm{~min}$. A maximum of six uniaxial accelerometers (ICsensors ${ }^{\mathrm{TM}} 5 \mathrm{~g}$ ) were mounted in varying directions on the trunk (sternum and shoulder) and the thighs and shanks. After initial evaluation, only the accelerometers mounted tangentially and radially in the sagittal plane on the sternum and tangentially in the sagittal plane on one thigh were further evaluated and will be discussed in this paper (Fig. 3). Not/all five subjects had all three of these accelerometers mounted. As stated, only the detection of the static or dynamic nature of activities and the discrimination of the dynamic activities were evaluated from this second set of experiments. To enable an unambiguous division of cycles in the analysis of the dynamic activities, membrane switches (special design for foot contact sensing in foot drop stimulators by Roessingh Adaptation Technique, Enschede, The Netherlands) were mounted under the heels and the balls of the feet to detect foot contact and clearance. These switches were used to divide the movements into cycles.

The subjects marked events (beginning and end of an activity) by a hand switch. The signals of the accelerometers and switches were recorded for later analysis by a portable measurement system (VTTAPORT ${ }^{\mathrm{TM}}$, McRoberts). The sampling rate was $100 \mathrm{~Hz}$. No presampling filters were used; the power spectra of the accelerometer signals were verified to be limited to a band width of approximately 10 $\mathrm{Hz}$. The accelerometer signals were preamplified (full scale was approximately $\pm 5 \mathrm{~g}$ ). The $\mathrm{AD}$ resolution varied with the number of channels used: 8,10 , or 12 bits, resulting in 25 levels to 400 levels for $1 \mathrm{~g}$. The lowest resolution did not compromise the detection, since it was in the same order as, or better than, the reproducibility of the accelerometer readings for static activities. The activities of the subjects were also recorded on video for subsequent timing and independent classification of the activities performed. Pressing of the event marker by the subject was combined with voice comments on the video.

\section{B. Detection of the Static or Dynamic Nature of Activities}

The tangential thigh accelerometer signal was chosen for the detection of the static or dynamic nature of activities because leg movements are pronounced in all investigated dynamic activities (walking, ascending stairs, descending stairs, cycling). The detection scheme of Fig. 2 was tested. High- and low-pass filters were both of the first order. Suitable cut-off frequencies were experimentally determined to be $0.5 \mathrm{~Hz}$ and $0.1 \mathrm{~Hz}$, respectively. The detector threshold level was experimentally set at $0.7 \mathrm{~m} / \mathrm{s}^{2}$.

\section{Distinguishing Static Activities}

All static activities for the first five subjects were identified according to the order of the protocol. The mean values of the accelerometer signals for all static activity segments were determined. The average and standard deviations per static activity and per sensor were also determined for each subject. From these results the discrimination ability of the system for static activities was assessed.

\section{Distinguishing Dynamic Activities}

The beginning and end of all segments of cyclical dynamic activity performed by the second group of subjects were determined using the marker signal. Clearly deviating first and last cycles were deleted from the accelerometer recordings. The type of activity was independently identified from the video recordings, which was synchronized with the recorded signals by time and marker signals. The beginning and end of the cycles in each segment were identified from the foot switch data for walking, ascending, and descending stairs. In this way, the discrimination of dynamic acitivities from the accelerometer signal morphology could be investigated independently from the question whether the beginning and ending of the cycles could be discriminated directly from the accelerometer signals. As an exception, cycles of cycling were discriminated from the accelerometer signals directly because foot switch data was not reliable for cycling. This was done by applying a threshold detector after low-pass filtering the thigh accelerometer signals (5th order Butterworth filter with a cut-off frequency of $1 \mathrm{~Hz}$ for slow and comfortable speeds and $2 \mathrm{~Hz}$ for fast cycling).

The average signal values, standard deviations and cycle durations for all cycles were evaluated for the accelerometer signals. Mean and standard deviations were subsequently determined to assess the value of these parameters for the discrimination of the activities. Discrimination between dynamic 
activities on the basis of signal morphology was investigated for the tangentially mounted accelerometer on the thigh, because this sensor gave a clear cyclical signal for all cyclical movements at the frequency of the total movement, in contrast to the trunk accelerometer which displayed the double frequency.

The cycles of each dynamic activity for each subject of the second group were divided into two sets: a template set and a test set. A template signal was constructed for each dynamic activity by averaging the cycle signals of the template set, after normalizing the duration of the cycles. This normalization was done by resampling the signals for each cycle to $N=100$ samples using linear interpolation between samples. Discrimination of the dynamic activities was evaluated by determining the maximum circular crosscorrelation coefficients between the single cycle signals from the test set and the templates. The circular cross-correlation coefficient $\rho_{x y}(m)$ of two signals $x$ and $y$ is defined as

$$
\rho_{x y}(m)=\frac{1}{N-1} \sum_{i=1}^{N} \frac{(x(i)-\bar{x})\left(y_{C}(i-m)-\bar{y}\right)}{s_{x} s_{y}}
$$

with $N=100$ being the number of samples during one cycle after resampling of the signals, $y_{C}(i-m)$ represented the signal $y$ shifted over the time $m$, with the last $m$ samples relocated at the beginning of the cycle (circular shift), $\bar{x}$ and $\bar{y}$ represented the sample averages, and $s_{x}$ and $s_{y}$ represented the sample standard deviations of signals $x$ and $y$.

The maximum value of the cross-correlation coefficients between the single cycle signals and the templates was determined, varying the time shift $m$ over the full range from 0 to $N-1$. These maximum values indicate differences of the signal morphology during various dynamic activities. One of the dynamic activities was identified from the others if this maximum cross-correlation coefficient exceeded a preset threshold value. The reasons for evaluating the crosscorrelation coefficients for all time shifts $m$ were twofold. First, the beginning and ending of the cycles could not be identified on the basis of the same foot switch information for all movements, and for cycling the cycles were identified directly from one of the accelerometer signals. Secondly, if the division of cycles is done on the basis of one of the accelerometer signals directly rather than on the basis of foot switch data, a well-defined beginning and ending of a cycle is no longer defined.

The detector performance based on signal morphology was evaluated as a function of the detection threshold value $v$ using the receiver operating characteristics (ROC) [11]. The ROC can be conceived as a parameterized curve with $v$ being the varying threshold parameter displaying the percentage of correctly detected cycles of a given dynamic activity (estimate of detection probability $\hat{P}_{d}(v)$ ) as a function of $v$ against the percentage of cycles of other dynamic activities which were incorrectly identified as this activity (estimate of the type two errors false alarm probability $\hat{\beta}(v)$ ) as a function of $v$

$$
\operatorname{ROC}(v)=\left(\hat{\beta}(v), \hat{P}_{d}(v)\right)
$$

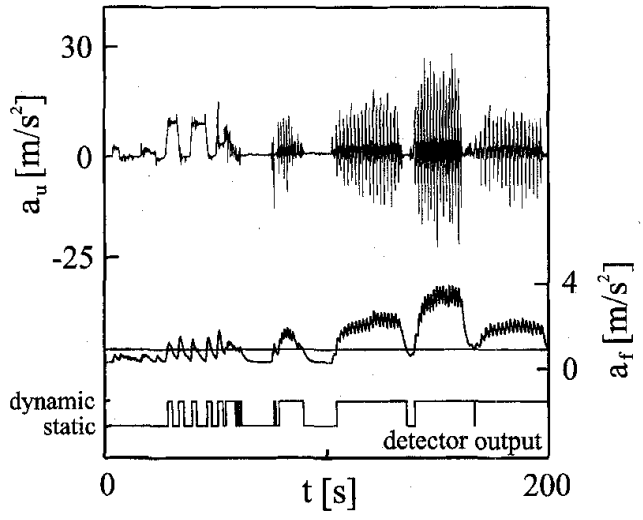

Fig. 4. Performance example for the detection of the static or dynamic characteristic of activities. The measured and processed signals $a_{u}$ and $a_{f}$, and the detector output are displayed. The signal of a thigh tangential sensor was used for the detection. High and low-pass filters were both of the first order. Their cut-off frequencies were $0.5 \mathrm{~Hz}$ and $0.1 \mathrm{~Hz}$ respectively. The threshold was set at $0.7 \mathrm{~m} / \mathrm{s}^{2}$.

\section{RESULTS}

\section{A. Detection of the Static or Dynamic Nature of Activities}

Fig. 4 shows a typical example of a tangential thigh accelerometer signal during static activities, short transitional movements and cyclical dynamic activities. The results indicate that it is possible to distinguish postures from movements in the proposed manner. Static segments, in which the signal does not change for several seconds are detected as static activity, and dynamic segments of several signal cycles are detected as dynamic. However, the output of the detector during short transitions between static segments, static segments of short duration and segments of slow movements depends on the detector settings. The cut-off frequency of the high-pass filter influences the detection of slow movements as either static or dynamic. The cut-off frequency of the low-pass filter influences the required duration of a static or dynamic segment to be detected and also determines the delay time between onset of the activity and its detection. The threshold setting determines above which level of movement an activity is called dynamic. The optimal setting of the detector parameters and subsequent performance evaluation requires explicit performance criteria. Development of such criteria, which may depend on the area of application, was not a topic in the current study. Therefore, the performance of the detector was not further analyzed. It should be noted that the evaluation of the methods for distinguishing static and dynamic activities as reported below did not rely on the detector of Fig. 2. Instead, static and dynamic segments of sufficient duration were independently selected for this evaluation using the marker signals, the video data, and the protocol order.

\section{B. Distinguishing Static Activities}

Fig. 5 depicts mean and standard deviation values of the quasistatic readings for the radial and tangential accelerometers on the sternum and tangential accelerometer on the 
thigh for the first group of five subjects. The radial sternum accelerometer [Fig. 5(a)] could distinguish sitting (SI) and standing (ST) from the other static activities: lying supine (LS), lying on the right side (LR), lying on the left side (LL) and lying prone (LP). The tangential sternum accelerometer [Fig. 5(b)] distinguished sitting, standing and lying on a side from either lying supine or prone. The tangential thigh accelerometer [Fig. 5(c)] distinguished standing and lying on either side from sitting, lying supine and lying prone. This accelerometer also distinguished lying prone from sitting and lying supine. The distinctions between static activities described above were all statistically significant within each subject (Wilcoxon rank-sum test, $\alpha<0.005$ ) and between subjects (Sign test, $\alpha<0.05$ ), evaluating all selected static segments of all five subjects (in total 150 measurements). In fact, all selected static segments were well classified by combining the radial sternum and tangential thigh accelerometer measurements, as shown in Fig. 6. Only lying on the left and right sides (LR, LL) could not be distinguished with these two accelerometers.

\section{Distinguishing Dynamic Activities}

Means and standard deviations of the mean signal values for walking (slow WS, comfortable WC, and fast speeds WF), ascending and descending stairs (SA and SD) and cycling (slow CS, comfortable CC, and fast speeds CF) are displayed in Fig. 7 for the radial and tangential accelerometers on the sternum and tangential accelerometer on the thigh. It should be noted that data in Fig. 7 are not complete for all of the second group of five subjects, since not all had the same sensor configuration or cycled with the considered configuration. The mean signal values of the sternum tangential accelerometer [Fig. 7(b)] and the thigh tangential accelerometer signal [Fig. 7(c)] both showed a statistically significant distinction between cycling (CS, CC, CF) and the other cyclical movements (Wilcoxon rank-sum test, $\alpha<$ 0.001 ) for each of the subjects tested. This can be ascribed to a difference of average orientation of the trunk and the thigh during cycling. However, intersubject significance of these findings could not be established because only two of the subjects had a sternum tangential accelerometer and only three subjects had a thigh tangential accelerometer. In contrast to the tangential sternum accelerometer, the radial sternum accelerometer did not show the distinction between cycling and the other cyclical movements in the two subjects in which it was measured [Fig. 7(a)]. This can be explained by the fact that the accelerometer in tangential direction is more sensitive for variation in average trunk orientation when the trunk is nearly vertical than the radial accelerometer [see (3)].

For each subject, the mean values of the thigh tangential accelerometer signals [Fig. 7(c)] showed a statistically significant distinction between ascending or descending stairs (SA and SD) and walking (WS, WC, WF) (Wilcoxon rank-sum test, $\alpha<0.001$ ). This finding was also statistically significant on an intersubject level (five subjects, sign test, $\alpha<0.05$ ). However, the inter-subject differences were such that a single detection threshold value for all subjects could not be used.

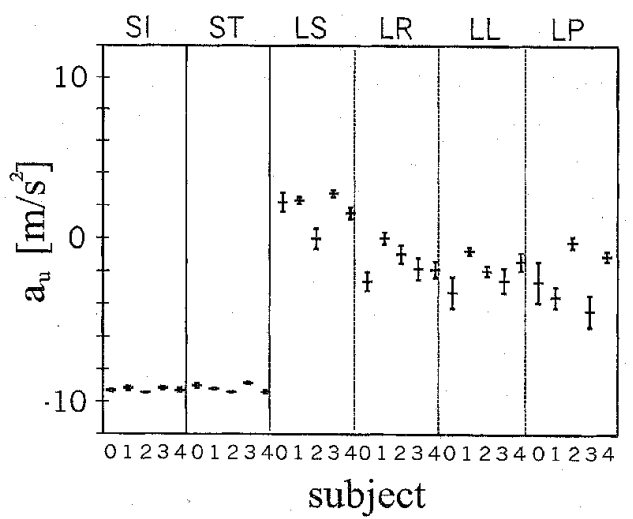

(a)

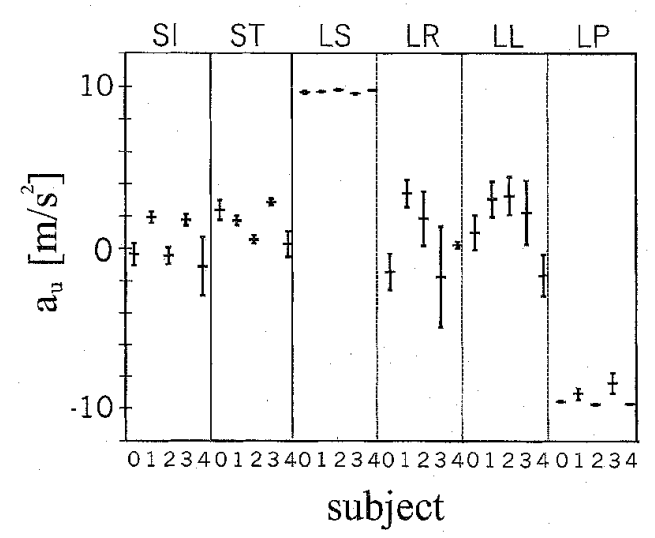

(b)

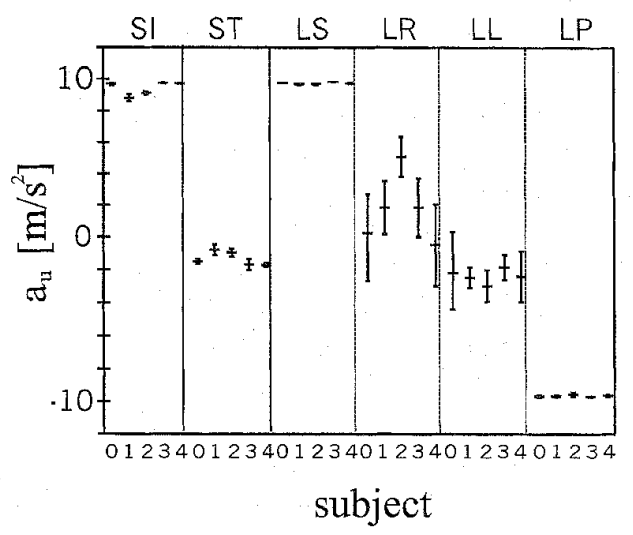

(c)

Fig. 5. Mean signal values ( \pm standard deviation) of the uniaxial accelerometers mounted radially on the sternum (a), tangentially on the sternum (b), and tangentially on the thigh (c) during the static activities sitting (SI), standing (ST), lying supine (LS), lying on the right side (LR), lying on the left side (LL), and lying prone (LP) for the first group of five subjects numbered $0-4$.

Means and standard deviations of the cycle durations and standard deviations of the signals are shown in Fig. 8 for the tangentially mounted accelerometer on the thigh. Both quantities are significantly correlated for walking and cycling $(\alpha<0.001)$. The correlation coefficients were -0.84 for walking and -0.73 for cycling. These correlation coeficients were determined after pooling the data for all subjects and for all speeds of walking respectively cycling. These results 


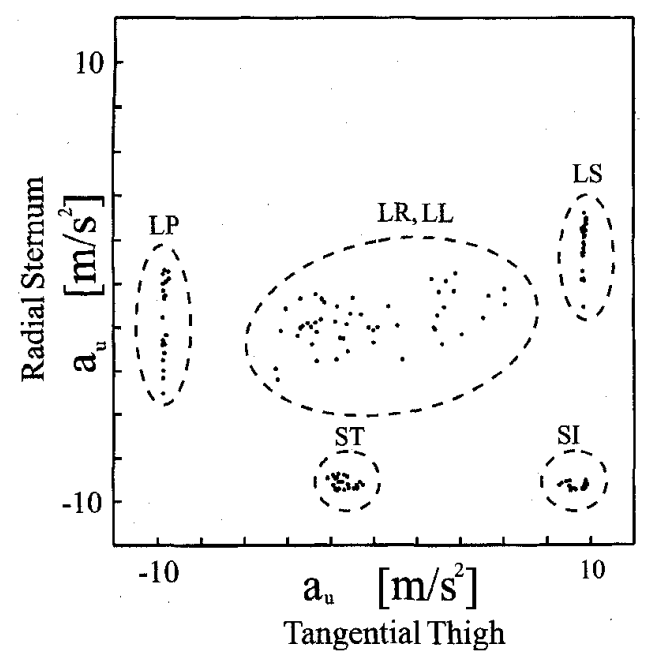

Fig. 6. Representation of the static activities sitting (SI), standing (ST), lying supine (LS), lying on the right side (LR), lying on the left side (LL), and lying prone (LP) in the plane spanned by the tangential thigh accelerometer signal (horizontal axis) and the radial sternum accelerometer (vertical axis). All selected segments for the first group of five subjects were pooled together in this figure.

indicate that cycle durations and standard deviations of the signal of the tangential thigh accelerometer contribute to the distinction of the speeds of walking and cycling. However, they do not contribute to the distinction of different kinds of dynamic activities.

Distinction of dynamic activities by morphological comparison of signals was investigated for the tangential thigh accelerometer. An example of the average cycle signals and standard deviations for walking at normal speed, ascending stairs and descending stairs are displayed in Fig. 9. The averages were taken over all identified cycles in the experiment. The signals for ascending and descending stairs varied more than for walking as indicated by the standard deviations.

The distinction in dynamic activities by signal morphology of individual cycles was investigated by determining the maximum correlation coefficients of individual cycles with templates from the different activities. Mean and standard deviation values of these correlation coefficients are given in Table I. The cross-correlation coefficients of individual cycles with templates of walking at any speed were significantly different from the cross-correlation coefficients of individual cycles of ascending or descending stairs with the same templates (Wilcoxon rank-sum test, $\alpha<0.001$ ). This indicates a good distinction between walking at any speed and ascending or descending stairs on the basis of accelerometer signal morphology. The cross-correlation coefficients of individual cycles of stair ascent with the templates for stair ascent or descent were also significantly different from these coefficients for individual cycles of stair descent (Wilcoxon rank-sum test, $\alpha<0.001$ ).

The actual detection performance on the basis of correlation coefficients are indicated by the ROC-curves in Fig. 10 . Walking at any speed appeared to be well distinguishable from walking up stairs or down stairs (Fig. 10(a)), because high values of the detection probability estimate $\hat{P}_{d}$ are combined

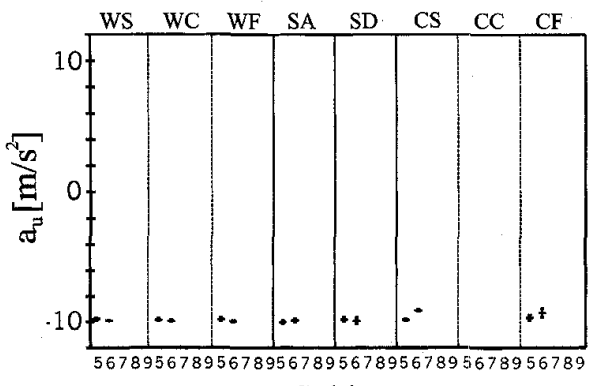

Subject

(a)

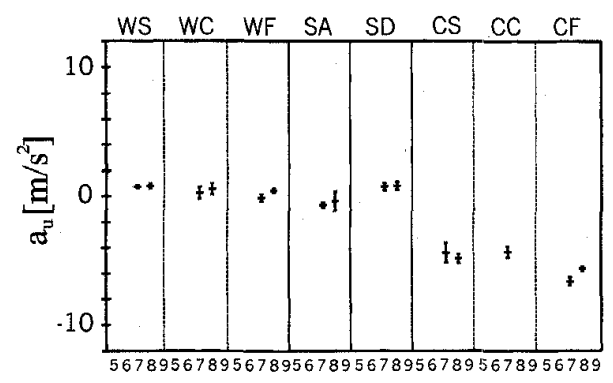

Subject

(b)

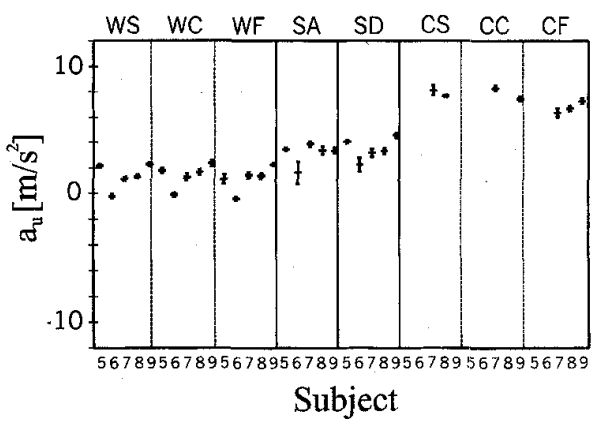

(c)

Fig. 7. Mean signal values ( \pm standard deviation) of the uniaxial accelerometers mounted radially on the sternum (a), tangentially on the sternum (b), and tangentially on the thigh (c) during the dynamic activities walking (at slow WS, comfortable WC and fast speeds WF), ascending stairs (SA), descending stairs (SD), and cycling (at slow CS, comfortable CC and fast speeds CF) for the second group of five subjects, numbered 5-9.

with low values of the type two error estimate $\hat{\beta}$. The estimated type one and type two errors $\left(1-\hat{P}_{d}\right.$ and $\hat{\beta}$, respectively) are equal at a threshold value of $v=0.33$ with an estimated overall detection error percentage of $2.5 \%$. Stair ascent and descent were less clearly distinguishable [Fig. 10(b)]: the estimated type one and type two errors are equal at a threshold value of $v=0.50$, with an estimated detection error percentage of $20 \%$, when correlating with the template for descending stairs. When correlating with the template for ascending stairs this estimated error percentage was $17 \%$.

\section{CONCLUSION AND DISCUSSION}

This paper describes a new approach to monitoring ambulatory activities for use in the domestic environment. The 
TABLE I

Maximal Correlation Coefficients (mean Values \pm Standard Deviation) for Single Cycle Signals from the Test Set and Templates of the Dynamic activities: Walking (at Slow WS, Comfortable WC and Fast SpeEds WF), Ascending Statrs (SA) and Descending Stairs (SD) For all Five Subjects of the SECond Group. The Signals Were all ObTaINed From

Thitgh Tangential accelerometers. The Templates Were Determined by Averaging the Cycles of the Template Set

\begin{tabular}{|c|c|c|c|c|c|c|}
\hline \multirow{2}{*}{$\begin{array}{l}\text { subject } \\
5 \\
\end{array}$} & \multirow{2}{*}{$\begin{array}{c}\begin{array}{c}\text { single cycles } \\
\text { from test set } \\
\text { (n cycles) }\end{array} \\
\end{array}$} & \multicolumn{5}{|c|}{$\begin{array}{l}\text { average cycle from template set } \\
\text { (n cycles) }\end{array}$} \\
\hline & & WS (4) & WN (18) & $\overline{W F(4)}$ & WU (6) & $\overline{W D(5)}$ \\
\hline & WS (4) & $090 \div 004$ & $0.74=0.06$ & $0,64 / 0.05$ & $0.67 \times 0.04$ & $0,35 \% 003$ \\
\hline & WN (15) & $0.81 \% 012$ & $0.85 \quad 014$ & $078 \quad 1014$ & $038 \quad 1008$ & $036 \quad 004$ \\
\hline & WF (3) & $0.68 \neq 0.07$ & $0.82-011$ & $077+013$ & 0500010 & $033 \quad 003$ \\
\hline & WU (6) & $0.50 \quad 0.11$ & $0.44 \quad 0.09$ & 0.4510 .05 & $0.56 \quad 013$ & $035 \% 0.05$ \\
\hline & WD (4) & $0.43<0.07$ & $0,41 \pm 0,06$ & $040 \quad 0008$ & $047 \approx 0,06$ & 0.540005 \\
\hline \multirow[t]{6}{*}{6} & & WS (5) & $\mathrm{WN}(42)$ & $W F(4)$ & WU (8) & WD (3) \\
\hline & WS (5) & 0.9310 .01 & 0701003 & $0,6910.03$ & 0.5610 .03 & $042 \quad 1006$ \\
\hline & WN (32) & $0,13 \pm 0.06$ & $091 \quad 1005$ & $0.85 \% 004$ & $0.38 \quad 0005$ & 056006 \\
\hline & WF (4) & 0.6910001 & $0,94 \quad 1003$ & 0931002 & $036 \quad 0001$ & 0.620004 \\
\hline & WU (5) & 0591006 & $044 \pm 004$ & $0.41+003$ & $055 \quad 006$ & $0.63 \quad 0004$ \\
\hline & WD (3) & 0451010 & 05810006 & $059 \angle 004$ & $044 \quad 0004$ & $028 \% 0 \mathrm{BS}$ \\
\hline \multirow[t]{6}{*}{7} & & WS (15) & WN (48) & WF (19) & WU (6) & WD (5) \\
\hline & WS (17) & $090+002$ & $088 \quad 0.04$ & $076 \% 0.06$ & $056<007$ & $043 \div 007$ \\
\hline & WN (43) & $0.78 \quad 0.07$ & 089940.07 & 0.8900 .08 & $054 \quad 00.09$ & $041 \% 005$ \\
\hline & WF (11) & $068 \quad 008$ & $0.83 \quad 0005$ & $089 \quad 005$ & $0.46 \quad 000$ & 0412006 \\
\hline & WU (4) & $0.59 \neq 003$ & 0.5400 .02 & $0.44 \pm 001$ & $0.88 \quad 0.03$ & $0.49 \quad 1000$ \\
\hline & WD (3) & $042 \quad 1000$ & $0.35 \div 0.07$ & 0310003 & $0.39 \geq 010$ & $0.72 \quad 0.05$ \\
\hline \multirow[t]{6}{*}{8} & & WS (9) & WN (52) & WF (7) & WU (46) & WD (3) \\
\hline & WS $(8)$ & 0881005 & 0771011 & $068 \quad 008$ & 0571005 & 0460002 \\
\hline & WN (49) & $073 \quad \square 0.06$ & $0.83 \neq 0.08$ & 007300.09 & $0.56 \$ 0.06$ & 0380006 \\
\hline & $\mathrm{WF}(6)$ & $0.66=0.06$ & $077 \times 0.08$ & $087<0.01$ & $0.45 \quad+0.04$ & $0,35 \propto 004$ \\
\hline & WU(18) & $054 \quad 009$ & 0551007 & 0491007 & 0581014 & 0.4410 .05 \\
\hline & WD (3) & $0.45<0.05$ & $0.46 \div 0,02$ & $046 \quad 005$ & $0.46 \div 612$ & 0.9410 .08 \\
\hline \multirow[t]{6}{*}{9} & & WS (15) & WN (37) & WF (7) & WU (8) & $\mathrm{WD}(27)$ \\
\hline & WS (13) & $0.85 \quad 0.07$ & $0.89 \bigcirc 005$ & $082+005$ & $0.46+0.03$ & $035+0,03$ \\
\hline & WN (32) & 0751010 & 0.82000 & $078,10.3$ & $040,00 s$ & $033 \quad 0.04$ \\
\hline & WF (6) & $0.71=0.06$ & $086 \simeq 005$ & 0.891005 & $0.34 \bigcirc 0.03$ & $031 \quad 0002$ \\
\hline & WU (7) & $034=0.03$ & $039 \quad 004$ & 00321005 & $0.76=0.07$ & $0.30 \quad 00.02$ \\
\hline & WD (22) & 03210,08 & $0.36 \div 0.09$ & $0.36+0.08$ & $0.30 \quad 10.05$ & 0.621014 \\
\hline
\end{tabular}

feasibility of distinguishing several static and dynamic activities (standing, sitting, lying, walking, ascending stairs, descending stairs, and cycling) using a small set of two or three uniaxial accelerometers placed on the trunk and thigh has been investigated under controlled circumstances. Accelerometers comply well with the requirements of an ambulatory system: they are small, light weight, easy to mount on the skin, and do not cross joints. However, these methods still need to be evaluated in the domestic environment when daily activities are performed without a strict activity protocol in healthy subjects as well as in subjects with motor disorders. This is currently under investigation [3].

It was illustrated that static activities could be distinguished from dynamic activities using the detector displayed in Fig. 2. Static and dynamic activities of sufficient duration (several seconds, several cycles) could be well detected. The detection of short static or dynamic activites and slow dynamic activities depended on the setting of detector parameters. A fullperformance evaluation and optimal settings of the detector parameters (bandwidths of high and low pass filters and threshold) of this (static/dynamic) detector requires criteria, for example, stating what amount of small movements are allowed within static activities, and when slow movements should be called static or dynamic. These criteria may depend on the area of application of the detector. If criteria cannot be clearly formulated, the detector performance should be compared to an independent classification by experts.

It can be concluded that the static activities standing, sitting and lying can be distinguished using one radial accelerometer on the sternum and one tangential accelerometer on the thigh. It should be noted that only unambiguous static activities were performed and evaluated in this study (sitting upright, lying horizontally, upright standing). If all possible postures occur, static activities need to be clearly defined. For example, what trunk angle range should be associated with sitting and what range is defined as lying. This would influence the classification of sitting in a comfortable chair when the trunk is not vertical.

The results in this paper indicate that cycling can best be distinguished from walking, ascending and descending stairs by the mean signal values of the tangential sternum and thigh accelerometers. However, the intersubject significance of this finding was not established, since it was only investigated in two subjects. Walking can be distinguished from ascending or descending stairs by the mean signal values of the tangential thigh accelerometer or by the signal morphology of single 


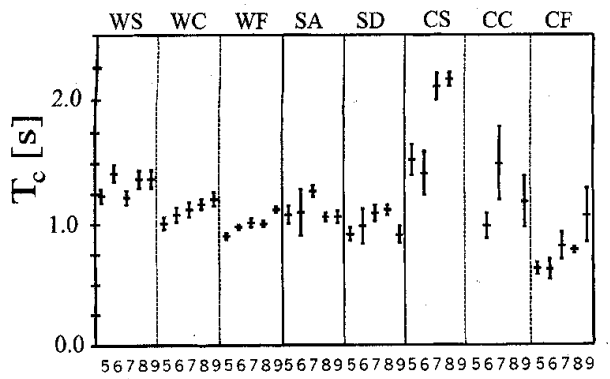

Subject

(a)

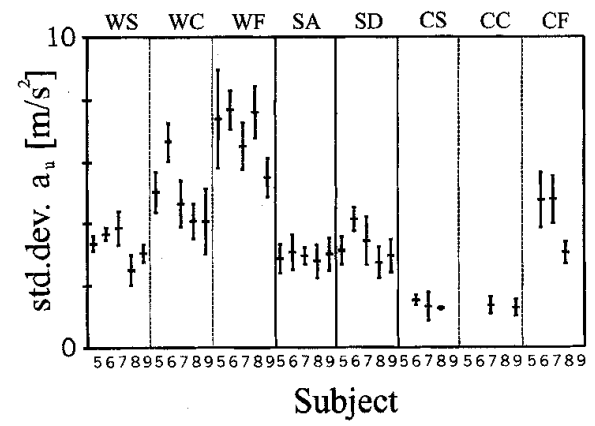

(b)

Fig. 8. Mean values ( \pm standard deviation) of the cycle durations (a) and standard deviations (b) of the signals of the uniaxial accelerometer mounted tangentially on the thigh during the dynamic activities walking (at slow WS, comfortable WC and fast speeds WF), walking up stairs (WU), walking down stairs (WD), and cycling (at slow CS, comfortable CC and fast speeds CF) for the second group of five subjects, numbered 5-9.

movement cycles, using the cross-correlation coefficient with templates. Intersubject significance was established for both methods. Ascending and descending stairs may be distinguished by signal morphology, although this discrimination is rather poor (estimated $20 \%$ erroneous classification). Speed of the dynamic activities walking and cycling can best be distinguished by cycle time or standard deviation of the cycles.

The performance of the dynamic activity classification on the basis of signal morphology may be improved if the average of more than one adjacent cycle is correlated with the templates of the different dynamic activities. These adjacent cycles are likely to belong to the same dynamic activity. Averaging these cycles may reduce the standard deviations of the correlation coefficients depicted in Table I and, therefore, improve the detection performance.

An accelerometer is most sensitive to inclination changes when its sensitive axis is horizontal [see (4)]. This should be taken into account if static or dynamic activities are to be distinguished on the basis of relatively small mean angle differences of body segments. As an example, cycling may be distinguished from walking, ascending and descending stairs using a tangential sternum accelerometer rather than a radially directed accelerometer on the same site.

In this study, the foot switch data was used to divide the segments of walking, ascending and descending stairs into cycles. Eventually, this division of cycles should be done

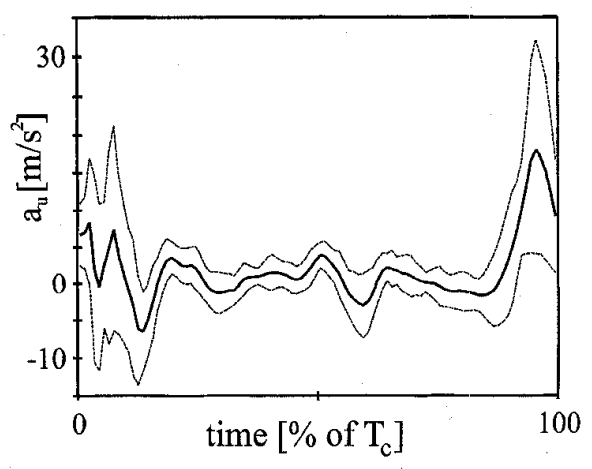

(a)

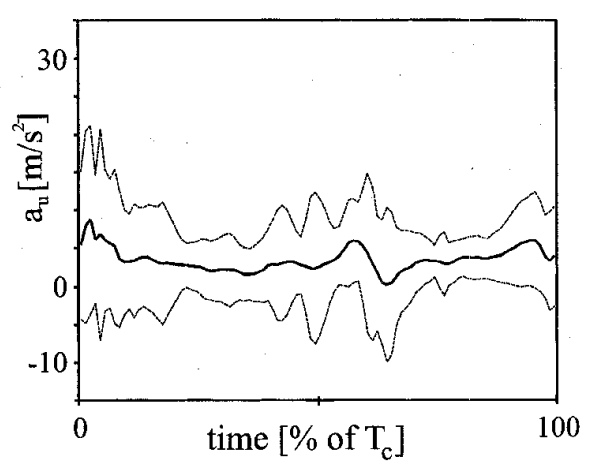

(b)

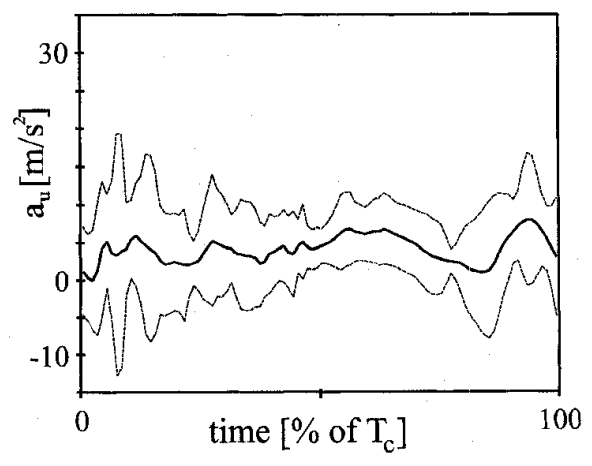

(c)

Fig. 9. Typical average cycle signals ( \pm standard deviation) of the uniaxial accelerometer tangentially mounted on the thigh for the dynamic activities walking at comfortable speed (a), ascending stairs (b), and descending stairs (c) (subject 5)

directly from the accelerometer signals, because applications in the domestic environment requires the smallest sensor set possible, positioned as closely as possible to the recorder, which is mostly worn at the waist. Preliminary investigations indicate that this division of the dynamic segments into cycles is feasible using bandfiltering with time-frequency analysis methods [8] or by determining maxima in the autocorrelation function.

Several applications of long-term monitoring of physiological quantities exist. Examples are the functional assessment of the cardiovascular system by long-term measuring heart rate, blood pressure and electrocardiography [12], and the assess- 


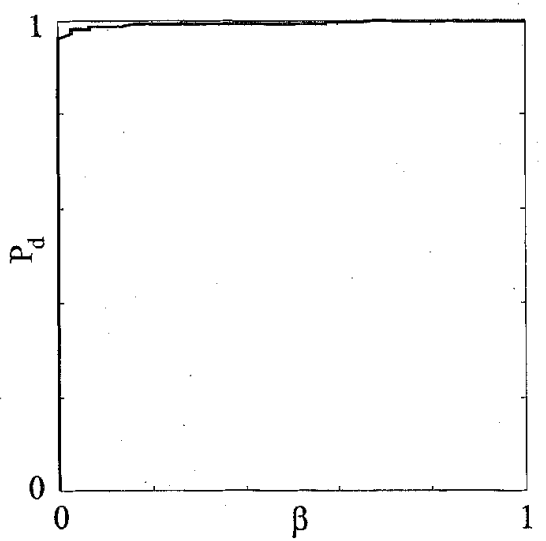

(a)

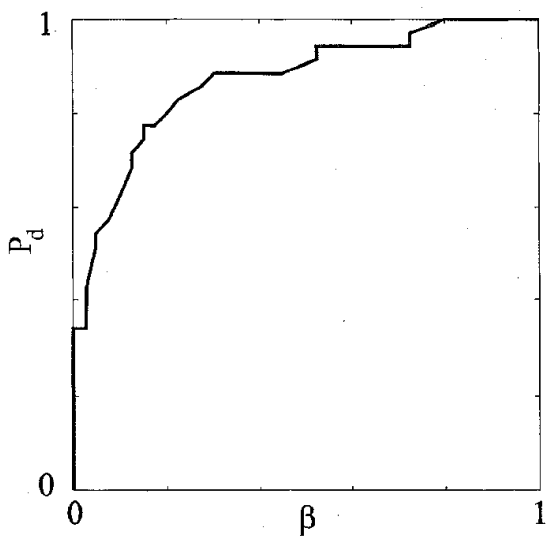

(b)

Fig. 10. Receiver operating characteristics (ROC) of dynamic activity detectors based on the maximal cross-correlation coefficients of the signals of individual cycles and templates of the dynamic activities. Signals of thigh tangential accelerometers were used for the detection. (a) Detection of walking at any speed out of a population of cycles of walking at any speed, ascending stairs and descending stairs $\left(n_{\mathrm{WS}, \mathrm{WC}, \mathrm{WF}}=248, n_{\mathrm{SA}, \mathrm{SD}}=75\right)$. For the detection, the maximal correlation coefficient was used with the templates for walking at slow (WS), comfortable (WC), and fast speeds (WF). (b) Detection of descending stairs out of a population of cycles of ascending and descending stairs, using the template for descending stairs $\left(n_{\mathrm{SA}}=40, n_{\mathrm{SD}}=35\right)$. All data of all dynamic activities of the second group of five subjects were pooled together in this figure. For each subject, the individual cycles were correlated with the template of that subject.

ment of spasticity by long-term measurement of electromyography [7]. Such long-term physiological measurements in the domestic environment may yield a more representative functional assessment than short-term measurements in the laboratory, since dysfunctions may occur infrequently or may be influenced by circumstances. In the applications of longterm measurements of physiological quantities, it is important to simultaneously record activities, since the occurrence of physiological dysfunction may be related to these activities. The methods for activity monitoring presented in this paper may be very suitable for this purpose.

\section{ACKNOWLEDGMENT}

The authors would like to thank R. Mayagoitia-Hill for helpful comments on the manuscript.

\section{REFERENCES}

[1] G. Baardman and A. Lissone, "Evaluation of the functionality of walking systems," in Advances in External Control of Human Extremities X, D. B. Popovic, Ed. Nauka, Belgrade: 1990, pp. 381-386.

[2] J. B. J. Bussmann, P. H. Veltink, F. Koelma, R. C. van Lummel, and H. J. Stam, "Ambulatory monitoring of mobility-related activities: The initial phase of the development of an activity monitor," European J. Phys. Medicine Rehab., vol. 5, pp. 2-7, 1995.

[3] J. B. J. Bussmann, P. H. Veltink, W. L. J. Martens, and H. J. Stam, "Activity monitoring with accelerometry," in Dynamic Analysis Using Body Fixed Sensors, P. H. Veltink and R. C. van Lummel, Eds., ISBN 90-9007328-0, 1994, pp. 12-16.

[4] P. E. Crago, H. J. Chizeck, M. K. Neuman, and F. T. Hambrecht, "Sensors for use with functional neuromuscular stimulation," IEEE Trans. Biomed. Eng., vol. 33, pp. 256-268, 1986.

[5] D. T. Davy and M. L. Audu, "A dynamic optimization technique for predicting muscle forces in the swing phase of gait," J. Biomechan., vol. 20 , pp. 187-201, 1987.

[6] V. T. Inman, H. J. Ralston, and F. Todd, Human Walking. Baltimore, MD: Williams \& Wilkins, 1981

[7] R. F. M. Kleissen, H. J. Hermens, Th. den Exter, J. A. de Kreek, and G. Zilvold, "Simultaneous measurement of surface EMG and movements for clinical use," Med. Biol. Eng., vol. 27, pp. 291-297, 1989.

[8] W. L. J. Martens, "Exploring the information content and some applications of body mounted piezo-resistive accelerometers," in Dynamic Analysis Using Body Fixed Sensors, P. H. Veltink and R. C. van Lummel, Eds., ISBN 90-9007328-0, 1994, pp. 8-11.

[9] J. R. W. Morris, "Accelerometry-A technique for the measurement of human body movements," J. Biomechan., pp. 729-736.

[10] A. J. Padgaonkar, K. W. Krieger, and A. I. King, "Measurement of angular acceleration of a rigid body using linear accelerometers," $J$. Appl. Mechan., vol. 42, pp. 552-556, 1975.

[11] I. Selin, Detection Theory. Princeton, NJ: Princeton University Press, 1965.

[1.2] J. H. M. Tulen, A. H. van den Meiracker, and A. J. Man in 't Veld, "Hemodnamic variability and locomoter activity: Clinical applications," Dynamic Analysis Using Body Fixed Sensors, P. H. Veltink and R. C. van Lummel, Eds., ISBN 90-9007328-0, 1994, pp. 73-77.

[13] R. W. Soames, C. D. Blake, J. R. R. Stott, A. Goodbody, and D. A. Brewerton, "Measurement of pressure under the foot during function," Med. Biol. Eng. Comput, vol. 20, pp. 489-495, 1982.

[14] P. H. Veltink, H. M. Franken, J. A. van Alsté, and H. B. K. Boom, "Modeling the optimal control of cyclical movements induced by functional electrical stimulation," Int. J. Artificial Organs, vol. 15, pp. $746-755,1992$.

[15] P. H. Veltink, H. B. J. Bussmann, F. Koelma, H. M. Franken, W. L. J. Martens, and R. C. van Lummel, "The feasibility of posture and movement detection by accelerometry," in Proc. 15th Annu. Int. Conf. IEEE-EMBS, San Diego, CA, Oct. 1993, pp. 1230-1231.

[16] A. Th. M. Willemsen, J. A. van Alsté, and H. B. K. Boom, "Real-time gait assessment utilizing a new way of accelerometery," J. Biomechan., vol. 23 , pp. $859-863$.

[17] A. Th. M. Willemsen, F. Bloemhof, and H. B. K. Boom, "Automatic stance-swing phase detection from accelerometer data for peroneal nerve stimulation," IEEE Trans. Biomed. Eng., vol. 37, pp. 1201-1208, 1990.

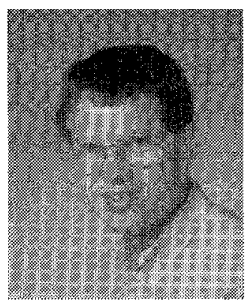

Peter H. Veltink (S'85-M'88) received the M.Sc. degree in electrical engineering from the University of Twente, Enschede, The Netherlands, in 1984 and the Ph.D. degree in 1988 in the topic of recruitment of myelinated nerve fibers during artificial nerve stimulation.

In 1989, he was a Visiting Assistant Professor at Case Western Reserve University, Cleveland, $\mathrm{OH}$ where he did research in the area of nonlinear control of muscle contraction. Currently, he is a Faculty Member of the Biomedical Engineering Department of the Faculty of Electrical Engineering, University of Twente. His research is in the area of control of functional neuromuscular stimulation and the application of body mounted sensors for human movement analysis. He works closely with the Research Department of the Roessingh Rehabilitation Center in Enschede. 


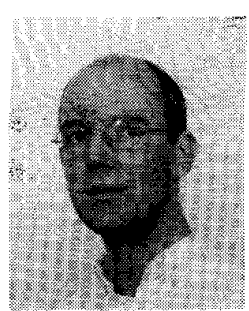

Hans B. J. Bussmann received the physical therapy degree in 1983 and the Master's degree in human movement sciences from Free University, Amsterdam, The Netherlands, in 1991.

Since 1992, he has been a member of the Faculty of Medicine and Health Sciences of the Erasmus University, Rotterdam, The Netherlands, in the Department of Rehabilitation Medicine. He is involved in the research project "ambulatory monitoring of the quantity, quality, and physical load of mobilityrelated activities." His main topic within this project is the development, validation, and use of an activity monitor.

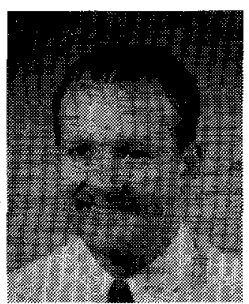

Wiebe de Vries was born in Kootstertille, the Netherlands, in 1967 . He received the electronic engineering degree from the HTS Leeuwarden, the Netherlands, in 1990 and the Ir. Degree in electrical engineering from the University of Twente, the Netherlands, in 1993.

From 1993 to 1995 , he was a Research Fellow at the Department of Biomedical Engineering, University of Twente. Presently, he is a Quality Control Engineer at the Research and Development Department of Drger Medical Electronics, where he is involved in the testing of patient monitoring equipment and the Conformity Assessment Procedures for the Medical Devices Directive (93/42/EEC).
Wim L. J. Martens received the M.Sc.E.E. degree from the University of Eindhoven, the Netherlands, on time-varying 'Wiener' filtering of evoked potentials.

After a study on echographic resolution enhancement via inverse filtering at the University of Limburg, Maastricht, he became the general Medical Physicist at Epilepsy Centre 'Kempenhaeghe,' Heeze, where he received his postdoctoral accreditation in 1984. He developed knowledge-based concepts for analysis of sleep and respiratory events. In 1990, he established PhyVision bv to develop the fast time frequency transform (FTFT), a novel concept for joint time-frequency analysis. He in now fully involved in developing applications for FTFT as capture of any kind of nonstationary event in the combined study of brain, respitatory, and cardiac functioning.

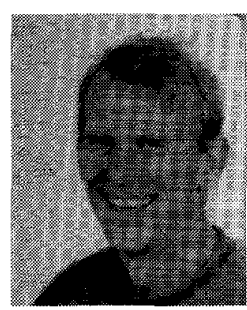

Rob C. Van Lummel has a background in physical education and sport and scholastic instruction With 20 years experience as a Sports Training and Kinematics Instructor, he established his own company focusing on human movement technology. He has initiated several international projects for the development of ambulatory monitoring methods for clinical applications based on biomechanical sensors (e.g., EUREKA projects DynaPort and Back Pain Monitor). His research interests are movement analysis, gait analysis, work demand and work hardening analysis, and the integration of kinematic and physiological systems. 